\title{
Evidence for Supermassive Black Holes in Active Galactic Nuclei from Emission-Line Reverberation
}

\author{
Bradley M. Peterson ${ }^{1}$ and Amri Wandel ${ }^{2}$
}

\begin{abstract}
Enission-line variability data for Seyfert 1 galaxies provide strong evidence for the existence of supermassive black holes in the nuclei of these galaxies, and that the line-emitting gas is moving in the gravitational potential of that black hole. The time-delayed response of the emission lines to continuum variations is used to infer the size of the line-emitting region, which is then combined with measurements of the Doppler widths of the variable line components to estimate a virial mass. In the case of the best-studied galaxy, NGC 5548, various emission lines spanning an order of magnitude in distance from the central source show the expected $V \propto r^{-1 / 2}$ correlation between distance and line width, and are thus consistent with a single value for the mass. Two other Seyfert galaxies, NGC 7469 and 3C 390.3, show a similar relationship. We compute the ratio of luminosity to mass for these three objects and the narrow-line Seyfert 1 galaxy NGC 4051 and find that that the gravitational force on the line-emitting gas is much stronger than radiation pressure. These results strongly support the paradigm of gravitationally bound broad emission-line region clouds.
\end{abstract}

Subject headings: galaxies: active — galaxies: quasars: emission lines — galaxies: Seyfert

\section{Introduction}

Since the earliest days of research on quasars and active galactic nuclei (AGNs), supermassive black holes (SBHs) have been considered to be the most likely driving power of the activity in these sources. Indeed, compact dark masses, probably SBHs, have been detected in the cores of many normal galaxies using stellar dynamics (Kormendy \& Richstone 1995). In the case of AGNs, however, direct detection of nuclear SBHs through stellar-dynamical methods has not been achieved on account of technical difficulties arising from the large surface brightness contrast

\footnotetext{
${ }^{1}$ Department of Astronomy, The Ohio State University, 140 West 18th Avenue, Columbus, OH 43210-1173 Email: peterson@astronomy.ohio-state.edu

${ }^{2}$ Racah Institute, The Hebrew University, Jerusalem 91904, ISRAEL Email: amri@frodo.fiz.huji.ac.il
} 
between the stellar component of the galaxy and the AGN itself on arcsecond and smaller angular scales. Nevertheless, there is good evidence for SBHs in AGNs. The strongest evidence to date has been the detection of Keplerian motions of megamaser sources in the Seyfert galaxy NGC 4258 (Miyoshi et al. 1995). Also, asymmetric Fe K $\alpha$ emission in the X-ray spectra of AGNs (e.g., Tanaka et al. 1995) may show the relativistic signature of an SBH, but this remains somewhat speculative as Fe K $\alpha$ reverberation signatures have not yet been observed (e.g., Reynolds 2000).

Perhaps the best method for measurement of the central mass in most AGNs is emission-line "reverberation mapping" which allows direct measurement of the size of the broad-line region (BLR) in these objects (Blandford \& McKee 1982; Peterson \& Netzer 1998). By careful monitoring of the variability of the UV and optical continuum and the light travel-time delayed response of the emission lines, one can determine the size of the line-emitting region, or more precisely, the distance from the central source at which the broad emission-line response is most significant. The broad emission lines are resolved in wavelength, and their widths reflect the Doppler motion of line-emitting gas. Assuming the velocities of the line-emitting gas are controlled by gravity, the combination of the size estimate (from reverberation mapping) and velocity (from the line widths) can be used to estimate the gravitational potential in which the gas moves, and thus deduce central black hole masses by the virial theorem $M \approx r \sigma^{2} / G$, where the line velocity width $\sigma$ is typically several thousands of kilometers per second and the size of the emission-line region $r$ is typically a few to tens of light-days.

SBH masses have been estimated using this method for about three dozen AGNs (Wandel, Peterson, \& Malkan 1999; Kaspi et al. 2000). However, whether or not the broad emission-line widths actually reflect virial motion remains an open question and is thus a key issue in AGN mass measurement (Richstone et al. 1998). While the relative response times for the wings of the line profiles reveal no strong signature of bulk outflow, there are still viable models with non-gravitationally driven cloud motions. However, if the kinematics of the BLR can be proven to be gravitationally dominated, then the reverberation results provide an even more definitive demonstration of the existence of SBHs than megamaser kinematics because the BLR is more than two orders of magnitude closer to the central source than the megamaser sources; the inferred SBH mass would dominate the stellar mass within the BLR even for the highest possible stellar densities.

Whether or not reverberation-based masses can be trusted is an important issue on account of a possible discrepancy between reverberation-based AGN SBH masses and stellar-dynamical normal-galaxy SBH masses. In the case of normal galaxies, the SBH mass appears to correlate with the galactic bulge luminosity, with the $\mathrm{SBH}$ to spheriodal bulge mass ratio $M_{\mathrm{BH}} / M_{\text {bulge }}=0.006$ (Magorrian et al. 1998; Richstone et al. 1998). In the case of AGNs, however, a significantly lower value of $M_{\mathrm{BH}} / M_{\text {bulge }}$ has been found (Wandel 1999), indicating either a real difference between active and normal galaxies, or that one or both of the methods of mass determination is somehow biased. We note, however, that other authors find lower values for non-active galaxies, $M_{\mathrm{BH}} / M_{\text {bulge }}=0.002-0.003$ (Ho 1999; Kormendy \& Ho 2000). This lower value, combined with 
the uncertainties in AGN virial masses (discussed below), may explain most of the discrepancy between the black-hole masses derived by stellar-dynamical and reverberation methods (Wandel 2000).

Here we expand on our argument (Peterson \& Wandel 1999) that the broad emission-line variability data demonstrate that the BLR kinematics are Keplerian, i.e., that the emission-line cloud velocities are dominated by a central mass of order of $10^{7-8} M_{\odot}$ within the inner few light days. This seems to hold for each of the three best-studied Seyfert 1 galaxies. We believe that this

strongly supports the hypothesis that SBHs reside in the nuclei of active galaxies, and underscores the importance of the reverberation method for determination of the masses of SBHs in AGNs.

\section{The Keplerian Size-Velocity Relationship}

Measurement of the central mass using the virial mass estimate from emission lines requires a demonstration that the kinematics of the line-emitting gas are gravitationally dominated. A correlation between the broad-line width and emitting-region size of the form $\sigma \propto r^{-1 / 2}$ is consistent with a wide variety of gravitationally dominated kinematics. It thus provides good evidence for such a dynamical scenario, although alternative pictures which contrive to produce a similar result may not be ruled out definitively.

In measuring the widths of the emission lines, it is important to include in the measurement only the part of the emission line that is actually varying. This can be difficult on account of contamination of the broad lines by emission from the narrow-line region or other non-variable (or slowly varying) components of the line and, in some cases, contamination from other broad lines. We circumvent this problem by using the numerous spectra obtained in the reverberation experiments to compute mean and root-mean-square (rms) spectra, and we measure the width of the emission features in the rms spectrum. While in many cases measurement of the width of each line from the mean spectrum gives a similar result (Kaspi et al. 2000), our procedure ensures that the emission features in the rms spectrum accurately represent the parts of the emission line that are varying, and for which the time delays are measured (Peterson et al. 1998). In each rms spectrum, we determined the full-width at half-maximum (FWHM) of each measurable line, with a range of uncertainty estimated by the highest and lowest plausible settings of the underlying continuum.

In Fig. 1, we show the width of the line in the rms spectrum plotted as a function of the distance from the central source (upper horizontal axis) measured by the emission-line lag $\tau$ (lower horizontal axis) for various broad emission lines in three different Seyfert 1 galaxies, NGC 5548, NGC 7469, and 3C 390.3. All of the data used here are publicly available on the International AGN Watch website円. In the case of the best-studied galaxy, NGC 5548, lines that are strongest

1 http://www.astronomy.ohio-state.edu/ agnwatch 
in highly ionized gas (e.g., He II $\lambda 1640$ and He II $\lambda 4686$ ) have the shortest response times (a few days) and the largest Doppler widths $\left(V_{\mathrm{FWHM}} \gtrsim 8000 \mathrm{~km} \mathrm{~s}^{-1}\right)$, and lines that are more prominent in less highly ionized gas (e.g., $\mathrm{H} \beta \lambda 4861$ and $\mathrm{C}$ III $] \lambda 1909$ ) have longer time delays (more than 10 days) and narrower widths $\left(V_{\mathrm{FWHM}} \lesssim 7000 \mathrm{~km} \mathrm{~s}^{-1}\right)$.

Each data point in Fig. 1 provides an independent measurement of the virial mass in each respective galaxy, $M=f r_{\mathrm{BLR}} \sigma_{\mathrm{rms}}^{2} / G$, where $r_{\mathrm{BLR}}=c \tau$, and we take $\sigma_{\mathrm{rms}}=\sqrt{3} V_{\mathrm{FWHM}} / 2$ (Netzer 1990). The factor $f$ depends on the details of the geometry, kinematics, and orientation of the BLR and is expected to be of order unity. Within the measurement uncertainties, all the emission lines yield consistent values for the central mass. A weighted fit to the relationship $\log \left(V_{\mathrm{FWHM}}\right)=a+b \log \tau$ yields $b=-0.44 \pm 0.05$ for the case of NGC 5548, consistent with the expected value $b=-1 / 2$, and a reduced $\chi_{\nu}^{2}=3.30$ (compared with $\chi_{\nu}^{2}=3.58$ for a forced $b=-1 / 2$ fit, which is also shown in Fig. 1). From a weighted fit to these data, we obtain $M=5.9 \pm 2.5 \times 10^{7} M_{\odot}$ for the central mass for the SBH in NGC 5548. The formal uncertainty reflects measurement uncertainties in the time lag and line width.

Moreover, we note that this mass is systematically uncertain by a factor of a few, on account of the uncertainty in the unknown factor $f$. As an illustration, we consider the masses obtained from two more detailed models of NGC 5548: if we use the Wanders et al. (1995) model for the C IV $\lambda 1549$ emission line (line-emitting clouds in circular orbits of random inclination, illuminated by a biconical beam), the rms line width and transfer function centroid match the observations best for a central mass of $M=2.4 \times 10^{7} M_{\odot}$. Alternatively, if we try to match the $\mathrm{H} \beta \mathrm{rms}$ line width and transfer function centroid with a disk model and reasonable photoionization model parameters (Ferland et al. 1992), then masses in the range $(1.4-6.0) \times 10^{7} M_{\odot}$ are obtained as the inclination is varied from $90^{\circ}$ to $\sim 30^{\circ}$.

Although NGC 5548 has by far the most detailed and highest-quality reverberation data, it is not the only AGN that shows the Keplerian relationship. We are able to derive reliable reverberation sizes and rms profiles for multiple emission lines in at least two more Seyfert galaxies, NGC 7469 (Wanders et al. 1997; Collier et al. 1998; Kriss et al. 2000) and 3C 390.3 (Dietrich et al. 1998; O'Brien et al. 1998), also shown in Fig. 1. In both cases the virial mass values calculated from each line are consistent with the same central mass (for NGC $7469, M=8.4 \times 10^{6} M_{\odot}$, $\sim 7$ times smaller than NGC 5548, and for 3C 390.3, $M=3.2 \times 10^{8} M_{\odot}, \sim 5$ times larger than NGC 5548). Together these three examples span a range of almost two decades in virial mass, a factor of three in line width and of five in BLR size, suggesting that Keplerian radial velocity profiles are common in a wide range of AGNs.

\section{Complications and Caveats}

These results are consistent with a surprisingly simple description of the BLR. At least to first order, the BLR gas appears to orbit the central source, possibly in a flattened geometry related 
to the accretion-disk structure, although ellipsoidal or spherical geometries cannot be excluded. The fact that the highest ionization lines have the smallest response times and the largest Doppler widths indicates that the BLR has an ionization-stratified structure. The $\mathrm{H} \beta$ response time in NGC 5548 is known to depend on the mean luminosity of the central source in the sense that the emission-line lag is longer when the continuum source is brighter (Peterson et al. 1999). We also see that when the emission-line lag is longer, the line width is smaller, apparently because we are seeing enhanced response from line-emitting gas that is farther away from the central source.

While some of the important general characteristics of AGN emission lines and their variability are accounted for with such a simple explanation, it is nevertheless clear that the actual situation is more complex. A responsivity map of each line would presumably reveal an extended and complex geometry, similar to what is seen in the spatially extended narrow-line regions.

The most important difficulty is that accurate measurement of the SBH mass depends on knowing the detailed geometry and orientation of the BLR, i.e., determining the factor $f$. For example, if the BLR is a flattened disk, the virial mass we deduce can be off by a factor of a few on account of the unknown inclination. Is there in fact evidence that geometrical effects might be important? We believe that there is. While the fits to the data above are consistent with the virial interpretation, there is significant residual scatter. The residual scatter in the relationship may be at least in part ascribable to somewhat different geometries of the line-emitting region at different radii, i.e., different values of $f$; indeed, it has been often argued that the high- and low-ionization lines arise in very different regions (Collin-Souffrin et al. 1988). A notable example in this regard is the narrow-line Seyfert 1 galaxy NGC 4051, in which the hydrogen Balmer lines might arise in a low-inclination (nearly face-on) disk but the high-ionization lines may arise in an outflowing wind above and below the disk (Peterson et al. 2000). At the other extreme, the Balmer-line profiles of 3C 390.3 are double peaked, which is the expected profile of a high-inclination disk. In any case, 3C 390.3 may have a somewhat atypical BLR structure, however, as the usual pattern of increasing lag with decreasing ionization level seems to be reversed in this object (i.e., C IV $\lambda 1549$ has a larger lag and narrower Doppler width than $\mathrm{H} \beta$, in contrast to the trend seen in every other object that has been studied). Interpretation of double-peaked line profiles rather problematic; in NGC 5548 and Akn 120, for example, the Balmer lines are sometimes double-peaked, but single-peaked or multiple-peaked at other times (Peterson, Pogge, \& Wanders 1999).

In spite of these uncertainties, the data at hand seem to rule out models that predict a distance-radial-velocity relationship that is significantly different from the Keplerian case. For example, in most outflow models, a wind or radiation pressure-driven acceleration is assumed. In this case the velocity should increase with radius, or at least remain constant. A constant velocity width over the radial range sampled by the data of the reverberation-mapped emission lines can be ruled out at the confidence levels of $\gtrsim 99.9 \%, \gtrsim 99 \%$, and $\gtrsim 95 \%$ for NGC $5548,3 \mathrm{C} 390.3$, and NGC 7469, respectively. 


\section{Mass vs. Luminosity}

In order to determine whether the BLR could be radiatively accelerated, we estimate the ratio of gravitational to radiation pressure. This can be formulated in terms of the well-known Eddington limit, the luminosity above which the radiation pressure exceeds the gravitational force on an ionized gas, $L_{\mathrm{Edd}}=4 \pi G M m_{\mathrm{H}} / \sigma_{\mathrm{T}}=3 \times 10^{11} L_{\odot}\left(M / 10^{7} M_{\odot}\right)$. We can further define the Eddington ratio $F_{\text {rad }} / F_{\text {grav }}=L / L_{\text {Edd }}$. We have seen how reverberation data on the broad lines can give us a reliable estimate for the central mass. If in addition the total continuum luminosity can be estimated, it is possible to calculate the Eddington ratio. We note, of course, that the Eddington limit applies to the case of spherical accretion, and that super-Eddington accretion rates can be achieved in other geometries. The calculation is nevertheless interesting in that we can use it to argue against the case of radiatively driven spherical outflow.

Most of the AGN energy is radiated in the far-UV band, in the so called "UV bump" or "big blue bump," of which only the long-wavelength tail is directly observable. A very crude estimate of the UV-bump flux can be made by multiplying the energy flux in the observable spectrum by a constant bolometric correction factor, but this does not take into account the differences between the spectra of different AGNs (for example, more luminous objects appear to have flatter UV spectra, possibly indicating a more prominent UV bump). A presumably more accurate estimate can be made by inferring the ionizing luminosity from the broad emission lines, since the broad lines are powered by the ionizing continuum. By comparing the BLR size derived from reverberation data for some particular emission line (we use $\mathrm{H} \beta$ ) to the $R \propto L^{1 / 2}$ scaling that is predicted by naive photoionization theory, one can estimate the ionizing continuum luminosity from the central source (Wandel et al. 1999). From the definition of the ionization parameter $U=L_{\text {ion }} / 4 \pi r^{2} \bar{E} n c$, where $\bar{E}$ is the average photoionizing photon energy, and $n$ the mean particle density in the line-emitting gas, the BLR radius $r$ can be calculated by using an estimate of $L$. Inverting this method and measuring $r$ independently by reverberation methods, we can derive an estimate for the ionizing luminosity

$$
L_{\mathrm{ion}} \approx 6 \times 10^{43}\left(\frac{E}{1 \mathrm{Ryd}}\right)\left(\frac{n}{10^{10} \mathrm{~cm}^{-3}}\right)\left(\frac{\tau}{10 \text { days }}\right)^{2} \operatorname{ergs~s}^{-1},
$$

where $\tau$ is the time lag for the $\mathrm{H} \beta$ emission-line response, and the normalization has been determined from the Seyfert 1 sample of Wandel et al. (1999). Since the ionizing luminosity represents a significant fraction of the bolometric luminosity, together with the virial mass this implies that $L / L_{\text {Edd }} \propto L_{\text {ion }} / M_{\text {vir }}$.

In Fig. 2, we show both $L_{\text {ion }}$ and the optical luminosity $L_{\text {vis }}$ versus $M_{\text {vir }}$ for the three objects discussed here. In order to extend the range in mass and spectral properties, we have also included new results (Peterson et al. 2000) on the narrow-line Seyfert 1 galaxy NGC 4051. With the exception of NGC 7469, the visual luminosity of these objects is of order $\sim 0.001 L_{\mathrm{Edd}}$ and $L_{\mathrm{ion}} / L_{\mathrm{Edd}} \approx 0.01$. Typically $L_{\mathrm{ion}}$ is larger than $L_{\mathrm{vis}}$ by a factor of 10-30 (Wandel et al. 1999). NGC 7469 seems to have an exceptionally short lag relative to its visual luminosity, which gives 
$L_{\mathrm{ion}}$ smaller than $L_{\mathrm{vis}}$ and a relatively high value of $L_{\mathrm{vis}} / L_{\mathrm{Edd}} \approx 0.03$. For NGC $4051, L_{\mathrm{ion}} \approx 0.1$, larger than $L_{\mathrm{vis}}$ by a factor of 100 (presumably indicating the harder UV spectrum and soft X-ray excess typical of narrow-line Seyfert $1 \mathrm{~s}$ ). We note that the ratio $L_{\text {ion }} / L_{\text {Edd }}$ could be even smaller for a low-inclination flattened geometry, in which case our derived values of $L / L_{\mathrm{Edd}}$ are lower limits.

The important things to note are that (1) in any case $L / L_{\mathrm{Edd}}$ is small enough for gravity to dominate even after a bolometric correction and allowing for the uncertainty in the reverberation mass estimate, and (2) not surprisingly, the relationship between $L_{\text {ion }}$ and $M_{\text {vir }}$ has less scatter than the relationship between $L_{\text {vis }}$ and $M_{\text {vir }}$.

BMP is grateful for support of this work by the National Science Foundation and NASA through grants AST-9420080 and NAG5-8397, respectively, to The Ohio State University. AW wishes to acknowledge the hospitality of the Department of Physics and Astronomy at UCLA and Stanford University during this work. We thank Dr. Alex Filippenko for a predictably helpful and insightful referee's report on this paper. 


\section{REFERENCES}

Blandford, R.D., \& McKee, C.F. 1982, ApJ, 255, 419

Collier, S., et al. 1998, ApJ, 500, 162

Collin-Souffrin, S., Dyson, J.E., McDowell, J.C., \& Perry, J.J. 1988, MNRAS, 232, 539

Dietrich, M., et al. 1998, ApJS, 115, 185

Ferland G.J., Peterson B.M., Horne K., Welsh W.F., Nahar S.N., 1992, ApJ 387, 95

Ho, L.C. 1999, in Observational Evidence for Black Holes in the Universe, ed. S.K. Chakrabarti (Dordrecht: Kluwer), p. 157

Kaspi, S., Smith, P.S., Netzer, H., Maoz, D., Jannuzi, B.T., \& Giveon, U. 2000, ApJ, 533, 631

Kormendy, J., \& Ho, L.C. 2000, in Encyclopedia of Astronomy and Astrophysics (Institute of Physics Publishing), in press (astro-ph/0003268)

Kormendy, J., \& Richstone, D. 1995, ARA\&A, 33, 581

Kriss, G.A., Peterson, B.M., Crenshaw, D.M., \& Zheng, W. 2000, ApJ, 535, 58

Magorrian, J., et al. 1998, AJ, 115, 2285

Miyoshi, M., Moran, J., Herrnstein, J., Greenhill, L., Nakai, N., Diamond, P., \& Inoue, E. 1995, Nature, 373, 127

Netzer, H. 1990, in Active Galactic Nuclei, R.D. Blandford, H. Netzer, \& L. Woltjer (Berlin: Springer-Verlag), p. 137

O'Brien, P.T., et al. 1998, ApJ, 509, 163

Peterson, B.M., Pogge, R.W., \& Wanders, I. 1999, in Structure and Kinematics of Quasar Broad Line Regions, ed. C.M. Gaskell, W.N. Brandt, D. Dultzin-Hacyan, M. Dietrich, and M. Eracleous, Astronomical Society of the Pacific Conference Series, Vol. 175, p. 41

Peterson, B.M., \& Wandel, A. 1999, ApJ, 521, L95

Peterson, B.M., Wanders, I., Bertram, R., Hunley, J.F., Pogge, R.W., \& Wagner, R.M. 1998, ApJ, 501,82

Peterson, B.M., et al. 1999, ApJ, 510, 659

Peterson, B.M., et al. 2000, ApJ, 542, in press

Reynolds, C.S. 2000, ApJ, 533, 811

Richstone, D., et al. 1998, Nature, 395, A14

Tanaka, Y., et al. 1995, Nature, 375, 659

Wandel, A. 1999, ApJ, 506, L39

Wandel, A. 2000, in Probing the Physics of Active Galactic Nuclei by Multiwavelength Monitoring, ed. B.M. Peterson, R.S. Polidan, and R.W. Pogge (San Francisco: Astronomical Society of the Pacific), in press 
Wandel, A., Peterson, B.M., \& Malkan, M.A. 1999, ApJ, 526, 579

Wanders, I., Goad, M.R., Korista, K.T., Peterson, B.M., Horne, K., Ferland, G.J., Koratkar, A.P., Pogge, R.W., \& Shields, J.C. 1995, ApJ, 453, L87

Wanders, I., et al. 1997, ApJS, 113, 69

This preprint was prepared with the AAS $\mathrm{LAT}_{\mathrm{E}} \mathrm{X}$ macros v4.0. 
Fig. 1. - Line width in the rms spectrum plotted as a function of the distance from the central source (upper horizontal axis) as measured by the emission-line lag (lower horizontal axis) for various broad emission lines in NGC 7469, NGC 5548, and 3C 390.3. The dashed lines are best fits of each set of data to the relationship $\log V_{\mathrm{FWHM}}=a+b \log c \tau$, and the best-fit slopes are $b=-0.61 \pm 0.35,-0.44 \pm 0.05$, and $0.41 \pm 0.15$ for the three galaxies, respectively. The solid line shows the best fit to each set of data for fixed $b=-1 / 2$, yielding virial masses of $8.4 \times 10^{6} M_{\odot}$, $5.9 \times 10^{7} M_{\odot}$, and $3.2 \times 10^{8} M_{\odot}$ for the three respective galaxies.

Fig. 2.- The luminosity versus virial mass relationship for four AGNs. Squares with error bars denote $L_{\text {ion }}$, with virial masses based on all the lines shown in Fig. 1. The filled circles represent $L_{\mathrm{vis}}$, with virial masses based on Balmer lines only, as by Wandel, Peterson, \& Malkan (1999) and Kaspi et al. (2000), with the error bars omitted for clarity. The lines show constant values of the Eddington ratio $L / L_{\text {Edd }}$ between 0.001 and 1 . 


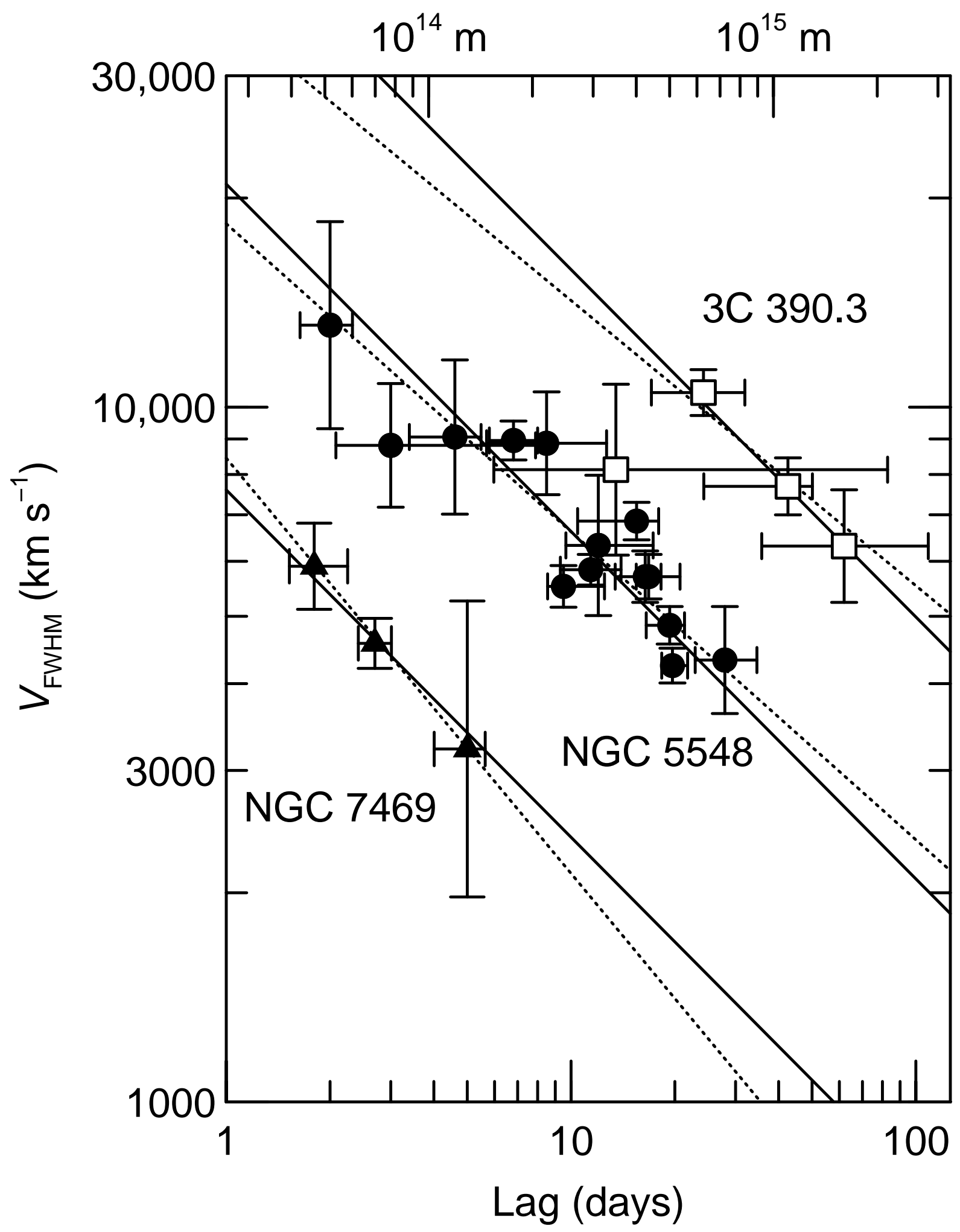




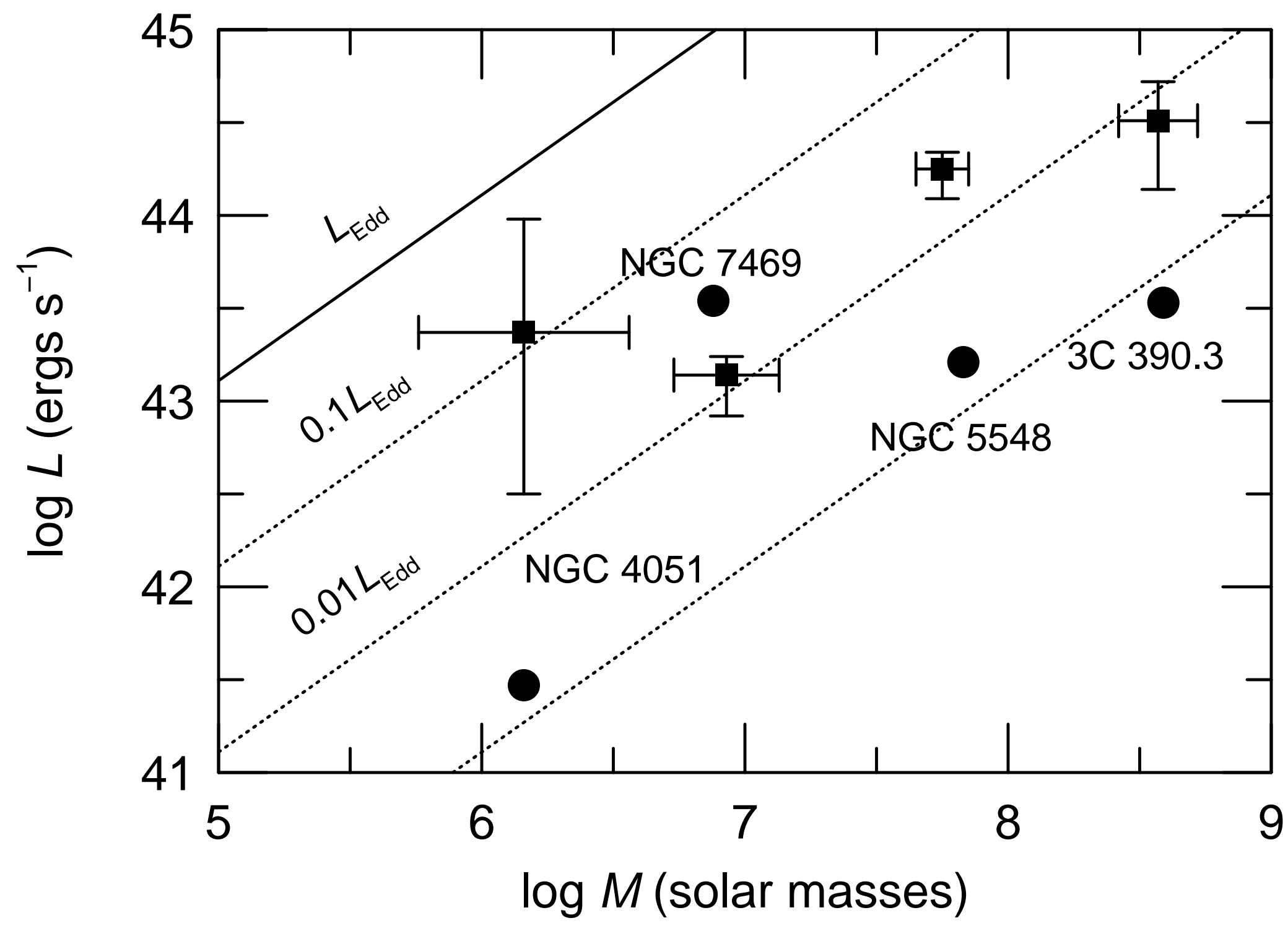

